\title{
Gastroenterites e infecções respiratórias agudas em crianças menores de 5 anos em área da região Sudeste do Brasil, 1986-1987. I - Infecções respiratórias agudas*
}

\section{Gastroenteritis and acute respiratory infections among children up to 5 years old in area of Southeastern Brazil, 1986-1987. I - Acute respiratory infections}

Rita de Cassia Barradas Barata, Eliseu Alves Waldman, José Cássio de Moraes, Ione Aquemi Guibu, Tatiana Rosov, Sueko Takimoto

Departamento de Medicina Social da Faculdade de Ciências Médicas da Santa Casa de Misericórdia de São Paulo. São Paulo, SP - Brasil (R.C.B.B., J.C.M., I.A.G.); Departamento de Epidemiologia da Faculdade de Saúde Pública da Universidade de São Paulo. São Paulo, SP - Brasil (E.A.W.); Departamento de Pediatria da Faculdade de Medicina da Universidade de São Paulo. São Paulo, SP - Brasil (T.R.); Instituto Adolfo Lutz. São Paulo, SP - Brasil (S.T.)

\begin{abstract}
Resumo
Estudou-se coorte constituída de uma amostra probabilística $(\mathrm{N}=468)$ de crianças menores de 5 anos, residentes em 5 áreas do Município de São Paulo, SP (Brasil), acompanhada durante 1 ano, por meio de entrevistas mensais. A pesquisa foi desenvolvida no período de março de 1986 a maio de 1987. Entre as características sociais e econômicas das famílias das crianças estudadas, estão: a) mediana da renda familiar "per capita" de um salário-mínimo da época; b) 29,3\% das crianças tinham pais migrantes com tempo médio de fixação no Município de São Paulo de 18,6 anos; c) 40\% das famílias utilizavam exclusivamente serviços de saúde públicos ou filantrópicos. Das crianças estudadas, $87,3 \%$ eram eutróficas; $94 \%$ haviam recebido todas as doses de vacina preconizadas pelo Programa Nacional de Imunizações; 90,6\% nunca haviam sido internadas em consequiência de infecção respiratória aguda (IRA). Durante a investigação foram identificados 554 episódios de IRA, com uma duração média de 6,8 dias, e uma incidência de 11,08 episódios por 100 crianças/mês. O grupo etário mais atingido foi o dos menores de 1 ano. Em 36,1\% dos casos de IRA identificados, verificaram-se eventos semelhantes no mesmo domicílio, sendo que em $53 \%$ desses episódios o caso-índice foi uma criança menor de 6 anos. Quanto ao tipo de atendimento, em 45,7\% dos episódios as crianças
\end{abstract}

* Pesquisa financiada pelo Conselho Nacional de Desenvolvimento Científico e Tecnológico/CNPq. Processo 40.0890/85 e pela Financiadora de Estudos e Projetos - Referência 43.85.01.00.

Correspondência para/Correspondence to: Rita de Cassia Barradas Barata - Departamento de Medicina Social da Faculdade de Ciências Médicas da Santa Casa de Misericórdia de São Paulo. Rua Dr. Cesário Mota Junior, 61 - 5º andar - 01221-020 São Paulo, SP - Brasil. Fax: (011) 222.0432 Edição subvencionada pela FAPESP. Processo 95/2290-6.

Recebido em 20.3.1995. Reapresentado em 31.3.1996. Aprovado em 24.5.1996. 
foram tratadas pelas próprias mães, 6,9\% recorreram a farmacêuticos, 46,7\% foram atendidas em diferentes tipos de ambulatórios e somente 4 casos $(0,7 \%)$ necessitaram tratamento hospitalar, com um deles evoluindo para óbito. As medidas terapêuticas mais utilizadas entre os casos que demandaram assistência médica foram a antibioticoterapia e os expectorantes. Alguns fatores socioeconômicos e antecedentes pessoais, tais como condições habitacionais, aglomeração intradomiciliar assim como antecedentes de doenças respiratórias, mostraram-se associados à incidência mais elevada de IRA.

Doenças respiratórias, epidemiologia. Fatores de risco.

\begin{abstract}
A probabilistic sample of children up to 5 years old was studied $(n=468)$. The epidemiological survey was carried out in five areas in the municipality of $S$. Paulo (Brazil), from March 1986 to May 1987. Data were obtained through household interviews once a month during one year. Some of the most relevant socioeconomic characteristics of the children's families are: low family income "per capita"; $29.3 \%$ of the parents were migrants with a mean period of residence in $S$. Paulo city of 18.6 years; $40 \%$ of the families had access exclusively to public or philanthropic health care services. Among the children included in the research, 87.3\% were eutrophic, 94\% were fully vaccined, 90.6\% had never been hospitalized as a consequence of acute respiratory infections (ARI). During the follow up of 1 year, 554 episodes of ARI with mean of 6.8 days of duration were identified. The incidence of ARI was 11.8 episodes per 100 children/month. The most vulnerable age group consisted of children up to one year of age. Thirty six percent of the ARI events were followed by at least one other case in the household. The index case in 53\% of these cases were children up to 6 years old. Around $52.6 \%$ of the episodes did not require a medical assistance, 46.7\% were assisted in ambulatory, 4 cases (0.7\%) have had hospital treatment and only one died. The most frequent therapeutical conduct regarding children submitted to medical care assistance was the prescription of antibiotics and expectorants. Some factors related to socialeconomic status and personal background such as living conditions and history of previous respiratory diseases were associated with higher incidence of ARI.
\end{abstract}

Respiratory tract diseases, epidemiology. Risk factors.

\section{INTRODUÇÃO}

As infecções respiratórias agudas (IRA) constituem uma das principais causas de morbidade em todo o mundo e de mortalidade nos países em desenvolvimento, onde os óbitos atingem principalmente as crianças. Estima-se que nesses países ocorram, anualmente, em menores de 5 anos, cerca de 4 milhões de mortes, a maior parte delas devido à pneumonia ${ }^{22}$.

A maior importância conferida às IRA nos países subdesenvolvidos está relacionada às infecções do trato respiratório inferior, cujas taxas de mortalidade são 10 a 15 vezes mais elevadas do que nas regiões industrializadas ${ }^{6,22}$.

As IRA constituem importante fator gerador de demanda de serviços de saúde em todos os países.
Estudos efetuados em áreas não industrializadas, verificaram que $10 \%$ a $15 \%$ das consultas ambulatoriais, para todas as faixas etárias, são motivadas por casos de IRA, esta cifra eleva-se para $30 \%$ a $35 \%$ em serviços ambulatoriais de pediatria. Das crianças que buscam serviços de saúde em virtude de acometimento por IRA, $70 \%$ a $80 \%$ delas enfrentam problemas do trato respiratório superior ${ }^{20}$.

A multiplicidade das formas clínicas, assim como, dos agentes envolvidos na determinação das IRA, dificultam sobremaneira a elaboração de estratégias simples e efetivas para seu controle ${ }^{1,4,5,10,15}$.

Na cidade de São Paulo, área em que foi desenvolvido o presente estudo, a taxa de mortalidade por pneumonia, em menores de 1 ano, vem caindo a partir de 1975, acompanhando a tendência da mortalidade infantil. No entanto, em termos relativos, supe- 
rou as diarréias, constituindo, desde o final da década de 70, a segunda principal causa de óbito nessa faixa etária. Em 1977, 1986 e 1993 as pneumonias foram responsáveis por, respectivamente, $23,2 \%$, $21,3 \%$ e $14,3 \%$ dos óbitos ocorridos em menores de 1 ano. Em 1993, constituíam a primeira causa de mortalidade em menores de 5 anos*.

Apesar da sua importância em saúde pública, são relativamente escassos no País, estudos populacionais, sejam eles longitudinais ou de corte transversal, relativos às IRA ${ }^{16,17}$.

O objetivo geral da presente pesquisa foi descrever as doenças diarréicas e as IRA em aspectos relacionados à magnitude e severidade do dano, à conduta terapêutica e demanda de serviços, por meio do seguimento de uma coorte de menores de 5 anos residentes em 5 áreas da cidade de São Paulo, com vistas a oferecer subsídios à elaboração de programas de controle, adequados à realidade do País. Os resultados serão divulgados em duas etapas, uma sobre infecções respiratórias agudas e outra sobre diarréia. O presente artigo, primeira etapa, terá por objetivos caracterizar as crianças e situá-las no contexto das condições de vida de suas famílias, descrever os aspectos do comportamento das IRA na população estudada, especialmente a duração, gravidade, evolução, tipo de atendimento e conduta terapêutica dos episódios registrados. Serão também estimadas a freqüência e a distribuição das IRA na população estudada, com especial atenção à incidência, diferenciais por faixa etária e por área de residência, casos secundários na família, sazonalidade e associação com alguns fatores socioeconômicos e ambientais.

\section{MATERIAL E MÉTODO}

\section{Área de Estudo}

A pesquisa foi conduzida nas seguintes áreas do $\mathrm{Mu}$ nicípio de São Paulo: subdistrito de paz da Barra Funda, bairros de Itaberaba e Vila Ramos, pertencentes ao subdistrito de paz de Nossa Senhora do Ó, e bairros de Vila Penteado e Parque Tietê pertencentes ao subdistrito de paz da Brasilândia.

A escolha dos bairros fundamentou-se em indicadores de saúde, demográficos e sociais obtidos em órgãos oficiais*, procurando selecionar populações com condições de vida diversificadas de modo a incluir crianças submetidas a diferentes estruturas epidemiológicas e fatores de risco para gastroenterites e infecções respiratórias agudas. A caracterização das áreas incluídas no estudo será apresentada com maiores detalhes na segunda etapa de publicação desta série.

\section{Amostragem}

O presente estudo abrangeu uma amostra probabilística dos domicílios situados nas áreas já mencionadas, compostas, segundo o censo demográfico de 1980 (Fundação $\left.\mathrm{IBGE}^{11}\right)$, por 92 setores censitários e 26.487 domicílios. Estabeleceu-se uma amostragem por área, de forma que, num primeiro momento os setores censitários constituíram as unidades amostrais para, em seguida, os domicílios constituírem as unidades amostrais elementares. A primeira etapa consistiu no sorteio de metade dos setores censitários, usando o critério de "um sim um não", para evitar que alguns segmentos da população fossem mais representados do que outros. Os resultados foram: 18 setores na Barra Funda, 6 no Parque Tietê e Itaberaba, 3 na Vila Ramos e 14 na Vila Penteado. Esses setores foram recenseados com os objetivos de atualizar o número de domicílios, nas áreas escolhidas, ao realmente existente em 1985, e de identificar aqueles que preenchessem o requisito de possuírem crianças de 0 a 4 anos, listando nominalmente aquelas situadas nessa faixa etária. Verificouse a existência de 8.712 menores de 5 anos. O passo seguinte foi o sorteio dos domicílios por meio de amostragem sistemática. Em todos os domicílios nos quais existiam mais de uma criança, na faixa etária de 0 a 4 anos, sorteou-se por processo casual simples apenas uma, a fim de se evitar replicações.

O estabelecimento do tamanho da amostra levou em consideração as disponibilidades de tempo, recursos humanos e financeiros, resultando numa amostra de 436 crianças $(5,0 \%$ da população).

Em virtude da duração da pesquisa, da alta mobilidade da população estudada e da possibilidade de recusas, optou-se por acrescentar 25,0\% de domicílios à amostra inicialmente estipulada. Foram finalmente sorteados 545 domicílios. Na amostra adotada foi respeitada a proporcionalidade quanto ao número de domicílios existentes em cada área, que ficaram assim distribuídos: 195 $(35,8 \%)$ domicílios na Barra Funda, $74(13,6 \%)$ no Parque Tietê, $63(11,6 \%)$ em Itaberaba, $39(7,1 \%)$ na Vila Ramos e 174 (31,9\%) na Vila Penteado.

\section{Processo Utilizado no Seguimento das Crianças}

As crianças sorteadas foram seguidas por meio de visitas domiciliares mensais, durante um ano, realizadas por estudantes do sexto ano do curso de medicina submetidos, previamente, a treinamento de um mês. Na primeira visita foi aplicado um questionário com o objetivo de obter informações relativas à composição familiar, situação socioeconômica, utilização de serviços de saúde, imunização, migração, condições da habitação e presença de animais domésticos.

Três meses após o início da aplicação desses questionários, supervisores do projeto tomaram uma amostra de $5 \%$ dos domicílios visitados e reaplicaram o questionário, 
verificando-se total consistência nas informações relativas à época do primeiro preenchimento do referido formulário.

A cada visita mensal, os entrevistadores forneciam uma cartela contendo 31 linhas correspondentes aos dias do mês, com o objetivo de aprimorar o recordatório da mãe ou responsável, visando a obter, principalmente, informações quanto ao início e fim de cada episódio, aos sinais e sintomas mais relevantes e à conduta terapêutica. Nessa visita, sempre que fosse registrado um episódio de IRA nos últimos 30 dias, era preenchido um questionário específico para essa intercorrência com informações referentes a sinais e sintomas, duração do episódio, conduta terapêutica, internação ou não, complicações e outros casos semelhantes na família.

Foi aplicado também um outro questionário para cada criança, abrangendo questões relativas à gestação e parto, alimentação, antecedentes mórbidos pessoais e familiares com ênfase para o aparelho respiratório e digestivo, hospitalizações anteriores e condição nutricional avaliada por meio de dados antropométricos e clínicos, cujo preenchimento ficou sob a responsabilidade de um médico pediatra, em visita domiciliar efetuada exclusivamente com essa finalidade.

\section{Definição de Caso}

\section{Infecção respiratória aguda:}

Infecção respiratória foi definida como a presença de um ou mais dos seguintes sintomas respiratórios: tosse, falta de ar, febre, dor de garganta, dor ou secreção do ouvido. O reaparecimento dos sintomas após 7 dias ou mais de interrupção, foi considerado como um novo episódio.

\section{Classificação das IRA}

Infecção de Vias Aéreas Superiores (IVAS): presença de tosse, coriza e febre sem comprometimento do estado geral e sem repercussões sistêmicas.

Gripe: tosse, coriza, febre, anorexia, mialgia e/ou outras repercussões sistêmicas.

Amigdalite/faringite: dor de garganta com ou sem febre, anorexia e mal-estar.

Otite média aguda: dor de ouvido com ou sem febre e secreção purulenta acompanhada de febre.

Bronquite: tosse produtiva, febre, falta de ar e "chiado no peito".

Broncopneumonia/pneumonia: taquipnéia, tosse, tiragem, batimentos nasais, cianose, falta de ar e febre.

\section{Conceitos e Categorias Utilizados}

Classes sociais: as famílias foram classificadas tomando como referência o esquema proposto por Singer ${ }^{21}$ e utilizado por Barros², em Ribeirão Preto.

Habitação: as condições de habitação foram avaliadas com fundamento na qualidade dos materiais utilizados na construção e, também, nas dimensões, insolação e presença ou não de umidade.
Imunização: as imunizações foram consideradas completas ou não, tomando por referência as normas do Programa Nacional de Imunizações. As imunizações foram consideradas incorretas quando o intervalo entre as doses ou a idade em que as vacinas foram aplicadas fugia aos padrões estabelecidos.

Antecedentes respiratórios da criança: pontuação de acordo com a importância: zero para nenhum antecedente; 1 ponto para episódios frequientes de IVAS, amigdalite, otite ou "chiado no peito"; 2 pontos para referência de "bronquites"; 3 pontos para pneumonia e 5 pontos para tuberculose.

Antecedentes familiares: pontuação de acordo com a importância: 1 ponto para asma; 2 pontos para tuberculose; 3 pontos para um dos pais fumantes; 4 pontos para consumo de até um maço por dia; 5 pontos para 2 maços/ dia; 6 pontos para mais de 2 maços/dia; 7 pontos para ambos os pais fumantes; 8 pontos para ambos os pais fumantes de até um maço por dia; 9 pontos para ambos os pais fumantes de dois maços por dia; 10 pontos para ambos os pais fumantes de mais de dois maços por dia.

Estado nutricional: avaliado a partir do peso, altura e sinais clínicos de desnutrição, utilizando o "software" Epi Info para classificação de dados antropométricos em percentis.

Os dados foram processados em microcomputador mediante a utilização do "software" Epi Info versão 6. Na análise estatística para testes de associação foram utilizados o teste $t$ para comparação entre médias e o qui quadrado. Para variáveis que apresentaram associação com a ocorrência de infecções respiratórias agudas foram calculados os riscos relativos e seus intervalos de confiança.

\section{RESULTADOS}

Das 545 crianças incluídas originalmente na amostra, 77 (14,1\%) haviam mudado de residência no intervalo de 6 meses transcorrido entre o recenseamento e o início do trabalho de campo. Portanto, foram efetivamente acompanhadas 468 crianças que apresentavam distribuição etária, por sexo e local de residência semelhante à amostra originalmente sorteada.

Os trabalhos de campo tiveram início em março de 1986, quando as 468 crianças ingressaram progressivamente no estudo e seu término ocorreu em maio de 1987. As perdas durante o período de acompanhamento foram de 82 crianças $(17,5 \%)$, sendo $70(15,0 \%)$ por mudança de endereço e $12(2,5 \%)$ por recusas. $\mathrm{O}$ número de visitas domiciliares mensais totalizaram 4.998, com 416,5 crianças/ano acompanhadas.

O tempo médio de seguimento variou entre as áreas de estudo. O menor tempo médio de acompanhamento foi observado na Barra Funda (9,8 meses) onde parte das famílias sorteadas mudou-se, logo no início da pesquisa, em função das desapropriações 
das residências. Na Vila Ramos, o tempo médio de seguimento foi de 10,5 meses; na Vila Penteado e no Parque Tietê 11,3 meses e em Itaberaba 11,5 meses.

\section{Caracterização das Famílias Estudadas sob o Aspecto Socioeconômico}

A classificação das famílias segundo estratos sociais, definida de acordo com a proposta de Singer ${ }^{21}$, mostrou grande concentração de crianças pertencentes ao proletariado $(66,8 \%)$ e à pequena burguesia $(19,2 \%)$. A distribuição das famílias, segundo estratos sociais, não mostrou diferenças estatisticamente significantes nos 5 bairros estudados.

Os resultados obtidos apontam a mediana da renda familiar entre 4 e 5 salários-mínimos (SM) da época. A mediana da renda familiar "per capita" correspondeu a apenas um SM, com 69,2\% das famílias com renda "per capita" igual ou menor do que um SM. A renda "per capita" média foi significantemente diferente $(\mathrm{p}<0,05)$ para os 5 bairros estudados. A menor renda "per capita" média foi observada entre as famílias do Parque Tietê (0,8 salários- mínimos) e a maior entre as famílias da Barra Funda (2,3 salários-mínimos).

Quanto às condições habitacionais que possivelmente podem influenciar o comportamento das IRA, elas eram adequadas para $55,9 \%$ das famílias estudadas, $41,1 \%$ das famílias habitavam construções em condições inadequadas de espaço, ventilação e iluminação e outras 3\% delas residiam em habitações precárias (barracos).

O número médio de pessoas por dormitório foi de 3,4 com mediana de 4. Em 15,8\% das famílias havia seis ou mais pessoas por dormitório. O número médio de pessoas por cama foi de 1,4 com mediana igual a 1 . Entretanto, em $2,7 \%$ dos domicílios havia três ou mais pessoas por cama.

Em 18,4\% e 29,9\% das crianças, respectivamente, ambos os pais e ao menos um deles eram nascidos no Município de São Paulo, em 29,3\% delas, ambos os pais eram originários de outros Estados. $\mathrm{O}$ tempo médio de fixação das famílias nesse município foi de 18,6 anos.

Com relação ao acesso a serviços de saúde, $40 \%$ das famílias utilizavam exclusivamente serviços públicos ou filantrópicos. A parcela que recorria, exclusivamente, a serviços privados era de apenas 7,5\%. Cerca de 1/3 das famílias utilizavam diversos tipos de serviços, entre públicos, privados e conveniados.

Quanto à utilização de serviços de saúde, conforme informação obtida à época da primeira visita domiciliar, $40 \%$ das famílias não haviam feito ne- nhuma consulta médica ou odontológica no último mês. Entre as demais, 24\% recorreram a uma consulta no último mês e $36 \%$ a duas ou mais.

Em termos de antecedentes mórbidos familiares, $16,0 \%$ negavam qualquer antecedente relacionado ao aparelho respiratório, porém $6,6 \%$ e $2,4 \%$ das crianças possuíam, respectivamente, casos de asma e tuberculose entre os familiares. No que tange ao tabagismo, $28,1 \%$ das crianças possuíam um dos pais fumante, enquanto que, em outros $12,8 \%$ delas, ambos apresentavam esse hábito.

\section{Características das Crianças Estudadas}

Houve predomínio do sexo masculino na amostra estudada $(53,8 \%)$, porém tal diferença não foi estatisticamente significante $(\mathrm{p}>0,05)$. Quanto à idade, $9 \%$ das crianças apresentavam cinco anos completos e somente $4,9 \%$ delas situavam-se na faixa de menores de um ano. Esse fato pode ser explicado pelo intervalo de seis meses decorridos entre a realização do processo de amostragem e o início de trabalho de campo. As crianças com 5 anos completos foram mantidas no estudo com objetivo de não alterar a amostra originalmente estabelecida.

Das 468 crianças estudadas apenas $24(5,1 \%)$ freqüentavam creches, número este reduzido, não permitindo verificar a possível associação dessa variável com uma incidência mais elevada de IRA.

De 309 crianças obtiveram-se informações a respeito das condições da gestação e do parto. Destas, $90,4 \%$ das mães haviam se submetido à assistência pré-natal, 94,8\% nasceram de gestações a termo; $47,5 \%, 47,2 \%$ e $5,3 \%$ dos partos foram, respectivamente, normais, por cesariana e por fórceps.

A mediana da duração do aleitamento materno foi de 2 meses, sendo que $25 \%$ das crianças nunca foram amamentadas. Com referência ao primeiro ano de vida, $60,7 \%$ delas receberam alimentação adequada para a idade, em termos da quantidade de itens incluídos na dieta e do momento de sua introdução; em 3,8\% das crianças, a alimentação foi inadequada segundo os mesmos critérios, restando $35,5 \%$ sem esta informação. De 309 submetidas a exame clínico, $87,3 \%$ eram eutróficas, $11,4 \%$ apresentavam desnutrição de primeiro grau e outros $1,3 \%$ desnutrição do segundo e terceiro grau.

A quase totalidade de crianças estudadas (94\%) havia recebido todas as doses de vacinas preconizadas pelo Programa Nacional de Imunizações, nas idades indicadas e com os intervalos corretos entre as doses. Das restantes, $4 \%$ apresentaram esquemas vacinais incompletos e $0,5 \%$ esquemas incorretos, 
isto é, sem respeitar os intervalos corretos entre as doses ou a idade mais indicada para vacinação. Apenas $1,5 \%$ das crianças não receberam vacinas por decisão das próprias mães.

Os antecedentes de doenças respiratórias, avaliados segundo critérios de frequiência e gravidade, apresentaram pontuações baixas, $54,4 \%$ ficaram situadas na faixa de até 2 pontos, 22,7\% entre 3 e 4 pontos e $22,9 \%$ com 5 pontos ou mais. Por esse motivo, que $90,6 \%$ das crianças nunca foram internadas, enquanto que $7,8 \%, 1,0 \%$ e $0,6 \%$ foram internadas, respectivamente, por 1,2 e 3 ou mais vezes. Com exceção do sarampo, a freqüência das doenças evitáveis por vacinação foi baixa, duas referiram difteria, oito coqueluche e uma poliomielite. No entanto, 35 haviam tido sarampo, sendo que 32 delas eram vacinadas (a questão do sarampo, pela sua importância, será discutida em outra publicação).

\section{Infecções Respiratórias Agudas}

Foram identificados 554 episódios de IRA nas 416,5 crianças/ano estudadas. A mediana da duração desses episódios foi de 5 dias, a média foi de 6,8 dias e o intervalo de confiança de 6,3 e 7,3 dias. Dos episódios estudados na amostra, 59,6\% deles duraram até 6 dias, 28,3\% entre 7 e 14 dias e em 12,1\% prolongaram-se por 15 dias ou mais (Tabela 1 ).

Tabela 1 - Duração dos episódios de infecções respiratórias agudas, em crianças de 0 a 5 anos, 1986 1987, Município de São Paulo.

\begin{tabular}{crcc}
\hline $\begin{array}{l}\text { Duração } \\
\text { (em Dias) }\end{array}$ & \multicolumn{2}{c}{ Episódios } & $\begin{array}{c}\text { Freqüência } \\
\text { acumulada } \\
(\%)\end{array}$ \\
\hline $1-3$ & № & $\%$ & 37,5 \\
$4-6$ & 122 & 22,1 & 59,6 \\
$7-9$ & 106 & 19,1 & 78,7 \\
$10-14$ & 51 & 9,2 & 87,9 \\
$15-19$ & 40 & 7,2 & 95,1 \\
$20-29$ & 15 & 2,7 & 97,8 \\
30 e mais & 12 & 2,2 & 100,0 \\
\hline Total & 554 & 100,0 & 100,0 \\
\hline
\end{tabular}

Os 554 episódios de IRA foram assim classificados com fundamento nas características clínicas conforme informações obtidas nas entrevistas domiciliares:

- Amigdalites (42 episódios): Apresentaram duração média de 4,2 dias com mediana de 2 dias. Duas crianças referiram eventos semelhantes na família. Apenas dois casos não receberam qualquer tipo de atendimento, os demais foram atendidos em hospitais, ambulatórios ou consultórios particulares.
Em 75\% dos casos a terapêutica foi com antibióticos. Nenhum deles necessitou de internação.

- Otite média aguda (30 casos): Para as otites, a duração média foi de 4,3 dias com mediana de dois dias. Quatro crianças referiram eventos semelhantes na família. Apenas três casos não receberam qualquer tipo de atendimento, os demais foram atendidos em hospitais, ambulatórios ou consultórios particulares. Em $76 \%$ deles o tratamento foi feito com antibióticos. Nenhum deles necessitou de internação.

- Infecções de vias aéreas superiores (411 casos): A duração média das IVAS foi de 5,9 dias com mediana de 5 dias. Cerca de $40 \%$ das crianças referiram eventos semelhantes na família havendo em média mais 1,6 pessoas afetadas. Em $37 \%$ dos casos as crianças recorreram a atendimento médico em hospitais, consultórios particulares ou ambulatórios de assistência primária. O tratamento mais utilizado foi o expectorante $(24,8 \%)$ seguido de antibióticos (24\%) e ácido acetil salicílico (20,7\%).

- Gripe (63 casos): A duração média dos casos classificados como gripe foi de 12,5 dias com mediana de 12 dias. Cerca de $45 \%$ das crianças atingidas tinham outros membros da família também doentes, havendo em média mais 1,9 pessoas afetadas. A maioria dos casos $(75 \%)$ foi atendida por médicos em hospitais, consultórios particulares ou serviços de assistência primária. Os tratamentos mais utilizados foram a antibioticoterapia $(52 \%) \mathrm{e}$ o uso de expectorantes $(20,6 \%)$.

- Broncopneumonia/Pneumonia (3 casos): Um dos casos apresentou 14 dias de duração, o outro 20 dias, o terceiro evoluiu para óbito com diagnóstico de broncopneumonia pós-sarampo. $\mathrm{O}$ atendimento desses casos foi feito em hospital e o tratamento, à base de antibióticos. Os dois primeiros casos receberam alta curados.

- Bronquite (6 casos): A duração média dos episódios foi de 5,2 dias, mas um dos casos permaneceu doente por mais de 15 dias. Dois casos foram atendidos por médicos e os demais pelas próprias mães. O tratamento mais utilizado foi a inalação seguida de uso de expectorante. Apenas 1 caso necessitou de hospitalização, recebendo alta curado.

Cerca de 36,1\% (200/554) dos episódios de IRA identificados nas crianças estudadas coincidiram com a ocorrência de outros casos na família. Em 4 casos de IRA da amostra, identificaram-se outras 5 pessoas residentes no mesmo domicílio, atingidas por agravo semelhante. Em 31\% (62/200) dos episódios em que além da criança estudada uma ou mais pessoas da família ficaram doentes, o caso-índice foi um dos pais, 
ou outro adulto residente no domicílio. As próprias crianças estudadas foram os casos-índice em 30\% (60/ 200) desses episódios, enquanto que, em outros $23 \%$ (46/200), o caso-índice foi um irmão com menos de 6 anos. Tem-se, portanto, que em $53 \%$ dos casos, os préescolares foram possivelmente os responsáveis pela introdução dessas infecções na residência. Os irmãos, em idade escolar (acima de 6 anos), foram casos-índice em apenas $16 \%$ dos episódios estudados.

Os 554 episódios de infecções respiratórias agudas geraram somente 4 internações hospitalares, sendo que um deles evoluiu para óbito em decorrência de broncopneumonia pós-sarampo. Os demais evoluíram para cura após tratamento ambulatorial ou mesmo na ausência de tratamento.

$\mathrm{O}$ tipo de atendimento buscado variou bastante. Em 45,7\% dos episódios não houve procura de nenhum tipo de atendimento, e em $6,9 \%$ das vezes as mães recorreram às farmácias. As crianças atendidas em serviços de saúde, ao todo $47,4 \%$ dos casos, distribuíram-se de modo equivalente entre hospitais, consultórios particulares e ambulatórios públicos de assistência primária. As condutas terapêuticas mais adotadas, entre os casos que procuraram serviços de saúde, foram a antibioticoterapia $(33,4 \%)$, expectorantes $(21,1 \%)$ e ácido acetil salicílico $(16,1 \%)$. Outras medidas tais como inalações, descongestionantes orais e broncodilatadores também foram utilizadas.

\section{Incidência}

A incidência de IRA nas crianças estudadas foi de 11,08 episódios por 100 crianças/mês de observação, com o intervalo de confiança variando de 9,64 a 11,7 episódios por 100 crianças/mês. A menor incidência de IRA foi observada na Barra Funda com o intervalo de confiança variando entre 7,08 a 9,68 e a maior em Itaberaba onde o intervalo de confiança da incidência situou-se entre 15,56 e 19,60, produzindo um diferencial estatisticamente significante $(\mathrm{p}<$ 0,01 ) de cerca de 2 vezes. (Tabela 2).

$\mathrm{Na}$ amostra, como um todo, $58 \%$ das crianças apresentaram episódios de IRA durante o período de acompanhamento, $42 \%$ delas tiveram 1 ou 2 episódios, $8 \%$ 3 episódios e os $8 \%$ restantes, 4 ou mais episódios. O número de episódios/ano de infecções respiratórias agudas, em cada bairro, variou de 1,92 a 2,78 episódios/ano por criança afetada (Tabela 2). A incidência das infecções respiratórias agudas variou com a idade, apresentando taxas significativamente mais altas em menores de 1 ano $(\mathrm{p}<0,005)$, declinando progressivamente nos outros grupos etários e voltando a subir no grupo de 5 anos (Tabela 3).
Tabela 2 - Episódios por crianças afetadas e incidência de infecções respiratórias agudas, em crianças de 0 a 5 anos, por bairro, 1986 - 1987, Município de São Paulo.

\begin{tabular}{lcc}
\hline Bairros & $\begin{array}{c}\text { Episódios por } \\
\text { criança/ano } \\
\text { afetada }\end{array}$ & $\begin{array}{c}\text { Incidência* } \\
\text { por } 100 \text { crianças/mês } \\
\text { (IC) }\end{array}$ \\
\hline Barra Funda & 1,92 & 7,08 a 9,68 \\
V.Ramos & 1,92 & 8,63 a 11,31 \\
Parque Tietê & 2,10 & 11,12 a 14,64 \\
V.Penteado & 2,12 & 9,53 a 11,95 \\
Itaberaba & 2,78 & 15,56 a 19,60 \\
\hline Total & 2,07 & 9,64 a 11,70 \\
\hline *(p<0,01) & & \\
IC: Intervalo de confiança & &
\end{tabular}

Tabela 3 - Incidência de infecções respiratórias agudas segundo a idade, 1986 - 1987, Município de São Paulo.

\begin{tabular}{ccc}
\hline & Idade \\
(anos) & Incidência/100 crianças/mês \\
\hline$<1$ & 19,28 \\
1 & 12,54 \\
2 & 10,25 \\
3 & 11,17 \\
4 & 9,61 \\
5 & 13,15 \\
\hline (p<0,005) & Total & 11,08 \\
\hline
\end{tabular}

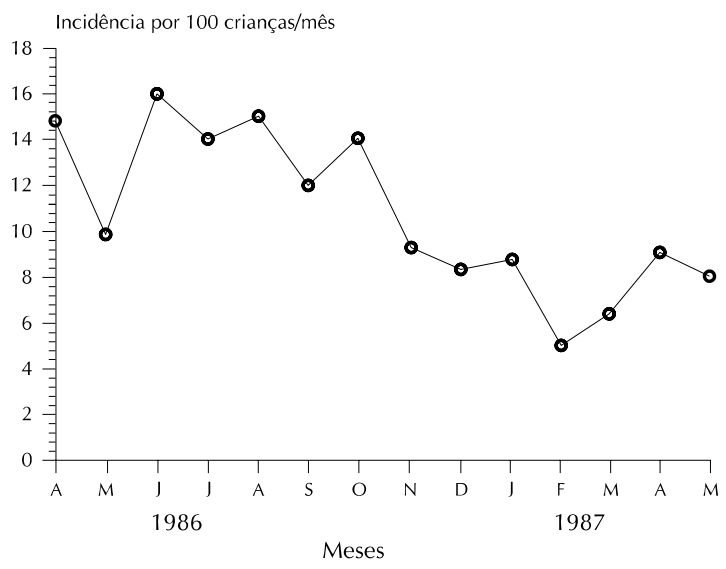

Figura 1 - Distribuição sazonal das infecções respiratórias agudas (incidência por 100 crianças/mês). São Paulo 19861987.

A distribuição mensal de infecções respiratórias agudas apresentou o comportamento esperado com incidências mais altas nos meses de inverno e mais baixas durante o verão (Fig.). Os valores foram significantemente mais altos em junho, agosto e outubro, ficando abaixo do esperado em janeiro, fevereiro e março $(\mathrm{p}<0,01)$. O comportamento das IRA foi semelhante em todos os bairros no que se refere à sazonalidade. 
A incidência de IRA não variou significantemente entre os estratos sociais ou entre os níveis de renda familiar "per capita", e no entanto, observou-se gradiente de incidência inversamente proporcional ao nível de grandeza dessas variáveis.

As associações de possíveis fatores de risco com a incidência de IRA entre as crianças estudadas constam na Tabela 4. Entre os fatores que apresentam associação têm-se as condições de habitação, onde as crianças residentes em habitações precárias apresentaram um risco relativo $(\mathrm{RR})$ de $1,14(\mathrm{P}<0,01) \mathrm{em}$ relação às que vivem em habitações adequadas; a aglomeração intradomiciliar, avaliada pelo número de pessoas por dormitório, apresentou RR de 1,34 $(\mathrm{P}<0,01)$ para aquelas que dormem com 5 ou mais pessoas no mesmo cômodo. A presença de animais domésticos no domicílio mostrou associação com a incidência de IRA com um RR de $1,17(\mathrm{P}<0,01)$ para as crianças cujas famílias possuíam gatos e cachorros. As crianças com antecedentes respiratórios e que receberam dois ou mais pontos em um critério, que combina freqüência e gravidade, apresentaram maior incidência de IRA com RR de 1,6 (P<0,01). Identificou-se também associação entre a incidência de IRA e desnutrição com RR de $1,11(\mathrm{P}<0,01)$ e entre desmame nos 6 primeiros meses de vida e a incidência de IRA em menores de 2 anos com RR de 1,21 ( $\mathrm{P}<0,01)$.

Tabela 4 - Fatores socioeconômicos e ambientais associados com a incidência de infecções respiratórias agudas, 1986-1987, Município de São Paulo.

\begin{tabular}{lcc}
\hline Fator & RR(bruto) & RR (ajustado) \\
\hline Aglomeração domiciliar & 1,40 & 1,34 \\
(5 pessoas ou + p/ dormitório) & & \\
Condições de habitação: & & \\
a) Adequada & 1,10 & $\mathrm{NS}$ \\
b) Precária & 1,20 & 1,14 \\
Animais domésticos & 1,50 & 1,17 \\
Baixo peso ao nascer & 2,00 & $\mathrm{NS}$ \\
Desnutrição & 1,11 & 1,11 \\
Antecedentes respiratórios & 2,00 & 1,60 \\
Desmame nos 6 primeiros & 1,24 & 1,21 \\
meses e IRA em < de 2 anos & & \\
Antecedentes familiares & 1,13 & $\mathrm{NS}$ \\
Pais fumantes & $\mathrm{NS}$ & $\mathrm{NS}$ \\
Renda familiar & $\mathrm{NS}$ & $\mathrm{NS}$ \\
Tempo de fixação em S. Paulo & $\mathrm{NS}$ & $\mathrm{NS}$ \\
$\quad$ (< ou = a 5 anos) & & \\
\hline NS: Não significante & & \\
P (<0,01) & &
\end{tabular}

\section{DISCUSSÃO}

A utilização de entrevistas domiciliares em investigações epidemiológicas constitui importante instrumento tanto para a identificação das necessidades sentidas pela população, geradoras de demanda a serviços de saúde, como para a obtenção de subsídios visando à caracterização do que se pode denominar de "necessidades não sentidas" no campo da saúde, cuja determinação é de grande importância no planejamento de programas ${ }^{7}$.

Analisando os resultados, vale comentar que as perdas ocorridas durante a pesquisa não determinaram uma diminuição expressiva do tamanho da amostra, sejam aquelas verificadas no intervalo entre o sorteio e o início do presente estudo $(14,1 \%)$, compensadas pelo acréscimo de $25 \%$ da amostra originalmente prevista, sejam as ocorridas durante o acompanhamento $(17,5 \%)$ pois estas se distribuíram regularmente no correr do inquérito, permitindo efetivamente o seguimento de 416,5 crianças/ ano, ou seja, uma amostra 4,5\% menor do que a originalmente estipulada (436 crianças). Quanto à representatividade, não houve sobreposição exata entre a amostra sorteada e aquela seguida, notando-se maior participação das crianças residentes em áreas com indicadores de saúde e demográficos mais favoráveis. Comparando a distribuição etária das crianças incluídas na amostra com a composição etária dos três subdistritos de paz estudados, observa-se menor proporção de crianças menores de um ano na amostra.

O nível de renda das famílias estudadas e as condições habitacionais, com somente $3 \%$ delas residindo em barracos, constituem indicações de que a amostra estudada apresenta condições de vida superiores à média encontrada no Município de São Paulo, em estudo realizado no período $1984 / 85^{17}$. O tempo médio de residência neste citado município $(18,6$ anos) sugerem situação de certa estabilidade social, apesar da proporção expressiva de migrantes.

A proporção elevada de famílias (36\%) que haviam se submetido a duas ou mais consultas médicas, no mês anterior à primeira visita domiciliar, sugere uma utilização excessiva de serviços de saúde, possivelmente relacionado a um grau insuficiente de resolutividade. Ainda em relação à saúde maternoinfantil, causam preocupação a elevada taxa de cesarianas $(47,2 \%)$ e a taxa de crianças nunca amamentadas ( $25 \%$ ), enquanto que a mediana da duração do aleitamento materno foi de 2 meses, situação pior do que a encontrada nos municípios de São Paulo e de Pelotas, mais ou menos na mesma época ${ }^{17,26}$. 
A duração média das IRA ( 6,8 dias) encontrada na presente pesquisa é pouco menor do que aquela mostrada na literatura ${ }^{15,18}$, possivelmente por refletir, ao menos em parte, a pequena gravidade dos casos identificados. Fortalece essa impressão o fato de $53,8 \%$ dos casos não demandarem serviços de saúde e somente 4 crianças necessitarem tratamento hospitalar, com um único óbito. Os casos de IRA classificados como broncopneumonia, bronquite, gripe e IVAS foram aqueles que geraram a maior proporção de consultas, enquanto que as broncopneumonias e as bronquites foram as únicas causas de internação, sendo que as broncopneumonias geraram 0,72 internações por criança/ano. Esta taxa de internação é pouco superior a encontrada por Foy ${ }^{10}$ nos EUA e muito inferior à verificada no Município de São Paulo em estudo realizado no período $1984 / 85^{17}$.

Os $36,1 \%$ dos episódios de IRA identificados refletiram possíveis surtos domiciliares com duas ou mais pessoas atingidas no domicílio o que pode sugerir a importante participação de vírus como agente etiológico. Por outro lado, os dados sugerem que em $69 \%$ destes prováveis surtos domiciliares, os responsáveis pela introdução da infecção no domicílio foram crianças, com predomínio dos préescolares.

A incidência de IRA encontrada é mais baixa do que as verificadas em estudos semelhantes para crianças menores de 5 anos, realizados em outras regiões do globo ${ }^{3,4,12}$, chegando a ser 6 vezes menor do que a incidência em crianças faveladas do Rio de Janei$\mathrm{ro}^{24}$. Tais valores refletem possivelmente as características particulares da população estudada, entre elas o fato de não incluir favelados, a menor representação das crianças de até um ano, a inclusão de crianças com 5 anos completos, a boa cobertura de prénatal, a elevada proporção de crianças adequadamente vacinadas, o pequeno número de desnutridos e de nascimentos de baixo peso, de crianças com antecedentes respiratórios e que freqüentam creches. Diferenças metodológicas também podem ter levado à verificação de uma incidência mais baixa, como por exemplo, o fato de ter-se estabelecido na definição de caso um intervalo de pelo menos 7 dias de interrupção dos sintomas para a caracterização de um novo caso.

A incidência de IRA encontrada no presente estudo foi cerca de 4 vezes maior do que a de gastroenterites na mesma coorte de crianças. Tal diferença situa-se no intervalo encontrado por Steinhoff e $\mathrm{John}^{23}$, em revisão sobre o comportamento desses dois agravos. Segundo esses mesmos autores, a incidência de IRA varia bastante de população para po- pulação, não parecendo haver um padrão determinado de distribuição em função do nível de desenvolvimento socioeconômico, diferentemente do que ocorre com as taxas de mortalidade causada pelas IRA que são maiores nas regiões subdesenvolvidas.

O diferencial verificado nas incidências por faixa etária obedecem o mesmo padrão encontrado em diversos relatos da literatura ${ }^{8,13,14,18,20,25}$. A variabilidade da incidência por faixa etária está condicionada, ao menos em parte, à situação imunitária do hospedeiro e ao predomínio de diferentes agentes infecciosos em grupos etários distintos ${ }^{10}$.

A ausência de informações etiológicas e o grande número de agentes possivelmente envolvidos dificultam considerações a respeito da sazonalidade encontrada, mas o predomínio dos casos de IRA nos meses de inverno sugerem participação importante da etiologia viral ${ }^{8,10}$.

A incidência das IRA na amostra estudada não variou significantemente entre os diferentes estratos sociais ou entre os níveis de renda familiar e "per capita", embora fosse observado um gradiente inversamente proporcional aos melhores níveis socioeconômicos. A composição da amostra levou à inclusão, em maior proporção, de crianças provenientes do proletariado, havendo relativamente poucas crianças da burguesia e do subproletariado, o que pode, em parte, ser responsável pela ausência de diferenciais marcantes. Outro fator a considerar é a possibilidade das mães, nos diferentes estratos, informarem melhor ou pior as ocorrências desses agravos. Monto e Ullman ${ }^{18}$ e Tupasi e col. ${ }^{25}$ relatam incidência mais elevada de IRA para as crianças de pior situação socioeconômica. Fleming e col. ${ }^{9}$ não encontraram relação entre incidências de infecções respiratórias agudas e renda familiar. $\mathrm{Na}$ análise desses dados devemos levar em conta, no entanto, que a baixa renda está freqüentemente associada a uma densidade maior de pessoas por domicílio e ao uso de roupas inadequadas ao clima, ambos fatores relacionados à maior incidência das IRA ${ }^{19}$.

A incidência de IRA entre crianças desnutridas e amamentadas por período inferior a 6 meses foi significantemente maior na amostra estudada se comparada com a encontrada em crianças que não apresentavam essas características, resultado que é semelhante ao descrito pela literatura ${ }^{4}$. A desnutrição deprime as defesas locais e sistêmicas contra as infecções respiratórias agudas, reduzindo a eficiência da barreira epitelial, a resposta imune e o reflexo da tosse. A deficiência nutricional e os episódios de IRA criam um ciclo vicioso que amplia os riscos de morte na infância ${ }^{22}$. 
As crianças estudadas e submetidas às condições habitacionais precárias, com maior aglomeração de pessoas dormindo no mesmo quarto e com antecedentes mórbidos do trato respiratório apresentaram, se comparadas com as não expostas a esses fatores, incidências mais elevadas de IRA. Estudos realizados em países não industrializados têm apontado a associação desses fatores com o aumento de pneumonias que requerem tratamento hospitalar ${ }^{22,25}$.

Os dados apontam a importância das IRA como causa de morbidade entre menores de 5 anos, pois no grupo estudado, apenas as IRA responderiam por cerca de 1 consulta/ano por criança estudada. Conside-

\section{REFERÊNCIAS BIBLIOGRÁFICAS}

1. AGUILAR ÁVILA, R. et al. Infección respiratoria aguda (IRA) en niños: incidencia, diagnóstico, etiologia, tratamiento y mortalidad. Diagnóstico, 18: 74-9, 1986.

2. BARROS, M.B.A. A utilização do conceito de classe social nos estudos dos perfis epidemiológicos: uma proposta. Rev. Saúde Pública, 20: 269-73, 1986.

3. BASHOUR, H.N.et al. A community-based study of acute respiratory infections among preschool children in Syria. $J$. Trop. Pediatr., 40: 207-13, 1994.

4. BERMAN, S. \& MCINTOSH, K. Selective primary health care: strategies for disease in the developing world. Rev. Infect. Dis., 7: 674-91, 1981.

5. BULLA, A. \& HITZE, K.L. Acute respiratory infections: a review. Bull. World Health Organ., 56: 481-98, 1978.

6. CAMARGO, P.A. et al. Mortalidade por pneumonia em crianças menores de 5 anos de idade em localidade do Estado de Minas Gerais (Brasil), 1979-1985. Rev. Saúde Pública, 23: 388-94, 1989.

7. CARVALHEIRO, J.R. Investigação epidemiológica e entrevistas domiciliárias. Rev. Saúde Pública, 15: 543-50, 1981.

8. DENNY, F.W. \& CLYDE, W.A. Acute lower respiratory tract infections in non hospitalized children. J. Pediatr., 108: 63546, 1986.

9. FLEMING, D.W. et al. Childhood upper respiratory tract infections: to what degree is incidence affected by day-care attendance. Pediatrics, 79: 55-60, 1987.

10. FOY, H.M. et al. Incidence and etiology of pneumonia, croup and bronchiolites in preschool children belonging to a prepared medical care group over a four years period. Am. J. Epidemiol., 97: 80-92, 1973. rando-se que o Município de São Paulo possuía, segundo o censo demográfico (Fundação IBGE ${ }^{11}$ ) de 1991, 988.381 crianças menores de 5anos e ainda que a amostra estudada não inclui população favelada e apresenta menor proporção de crianças abaixo de 1 ano do que a observada no município, pode-se estimar incidências ainda mais altas para o conjunto da população do município e uma taxa maior de casos graves, necessitando tratamento hospitalar. Por outro lado, o conjunto de informações obtidas pela presente pesquisa mostra que parte considerável dos casos poderiam ser adequadamente atendidos em serviços de assistência primária apresentando evolução favorável.

11. Fundação IBGE. Censo demográfico: dados distritais, São Paulo. Rio de Janeiro, 1982. (9ํㅡㄹ Recenseamento geral do Brasil, 1980). v. 1, t. 3, n. 17.

12. HERRERO, L. Respiratory infections in Central America. Pediatr. Res., 17: 1035-8, 1983.

13. JAMES, J.W. Longitudinal study of the morbidity of diarrhoeal and respiratory infections in malnourished children. Am. J. Clin. Nutr., 25: 690-4, 1972.

14. KAMATH, K.R. et al. Infection and disease in group of South Indian families. Am. J. Epidemiol., 89: 375-83, 1969.

15. LANG, T. et al. Acute respiratory infections a longitudinal study of 151 children in Burkina-Faso. Int. J. Epidemiol., 15: 553-60, 1986 .

16. MOLINA, M.C.B. et al. Nutritional status of children of urban low income communities Brazil. Rev. Saúde Pública, 23: 89-97, 1989.

17. MONTEIRO, C.A. Saúde e nutrição das crianças de São Paulo: diagnóstico, contrastes sociais e tendências. São Paulo, Hucitec-EDUSP, 1988.

18. MONTO, A.S. \& ULLMAN, B.M. Acute respiratory illness in an American community. JAMA, 227: 164-9, 1974.

19. MONTO, A.S. Studies of the community and family: acute respiratory illness and infection. Epidemiol. Rev., 16: 35173,1994 .

20. PIO, A. et al. Programa de la Organização Mundial de la Salud de infecciones respiratórias agudas en la infancia. Bol. Ofic. Sanit. Panam., 96: 283-95, 1984.

21. SINGER, P. Dominação e desigualdade. Rio de Janeiro, Paz e Terra, 1981. 
22. STANSFIELD, S.K. \& SHEPARD, S.D. Acute respiratory infection. In: Jamison,D.T. et al. Disease control priorities in developing countries. Oxford, Oxford University Press., 1993. p. 67-97, (Oxford Medical Publications).

23. STEINHOFF, M.C. \& JOHN, T.J. Acute respiratory infection of children in India. Pediatr. Res., 17: 1032-6, 1983.
24. SUTMOLLER, F. \& NASCIMENTO, J.P. Studies on acute respiratory infections in Brazil. Pediatr. Res., 17: 1038-40, 1983.

25. TUPASI, T.E. et al. Determinants of morbidity and mortality due to acute respiratory infections: implications for intervention. J. Infect. Dis., 157: 615-23, 1988.

26. VICTORA, C.G. Epidemiologia da desigualdade. São Paulo, Hucitec, 1988. 